\title{
Fundamental Limit of Plasmonic Cathodoluminescence
}

\author{
Franz-Philipp Schmidt,* Arthur Losquin, Michal Horák, Ulrich Hohenester, Michael Stöger-Pollach,
} and Joachim R. Krenn

Cite This: Nano Lett. 2021, 21, 590-596

Read Online

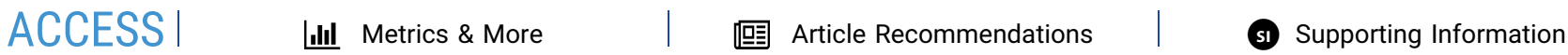

ABSTRACT: We use cathodoluminescence (CL) spectroscopy in a transmission electron microscope to probe the radial breathing mode of plasmonic silver nanodisks. A two-mirror detection system sandwiching the sample collects the CL emission in both directions, that is, backward and forward with respect to the electron beam trajectory. We unambiguously identify a spectral shift of about $8 \mathrm{~nm}$ in the CL spectra acquired from both sides and show that this asymmetry is induced by the electron beam itself. By numerical simulations, we confirm the observations and identify the underlying physical effect due to the interference of the CL emission patterns of an electron-beam-induced dipole

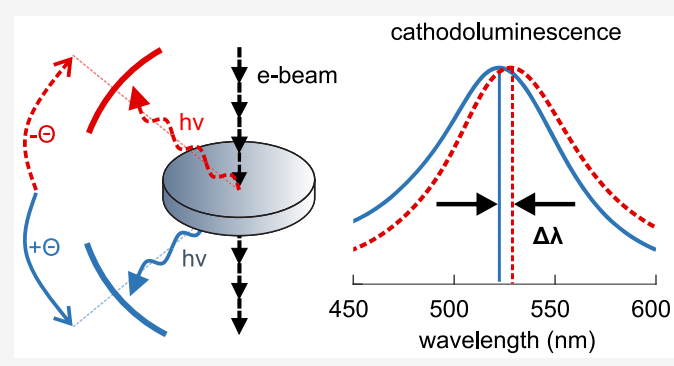
and the breathing mode. This effect can ultimately limit the achievable fidelity in CL measurements on any system involving multiple excitations and should therefore be considered with care in high-precision experiments.

KEYWORDS: Cathodoluminescence, transmission electron microscopy, plasmonics, radial breathing mode, nanoparticles.

$\mathrm{T}$ he insight in light-matter interaction at the nanoscale currently strongly benefits from developments in electron spectroscopy. In particular, electron energy loss spectroscopy (EELS) and cathodoluminescence (CL) in combination with scanning transmission electron microscopy (STEM) provide high spatial and spectral resolution. ${ }^{1-4}$ Of equal importance, advances in the theoretical understanding enable us to relate electron-based signals to local optical properties. ${ }^{5}$ Because of their strong optical effects on the nanoscale and the related rich physical phenomenology, plasmonic nanostructures are a prominent research field in STEM-based electron spectroscopy. Fast electrons, focused to a small spot, can excite plasmon modes on a local scale. Measuring electron energy loss in EELS yields information on both radiating and nonradiating plasmons, ${ }^{6-9}$ whereas CL only probes radiative excitations, that is, modes coupling to light. ${ }^{10-15}$

An intriguing effect from single plasmonic nanoparticles is directional light scattering. Upon excitation with light, scattering directionality is observed from geometrically asymmetric nanoparticles when different plasmon modes are excited within a narrow spectral range. The shape asymmetry can then lead to angle-dependent interference of the light fields scattered by different plasmon modes. ${ }^{16}$ In a STEM, this can be achieved even with symmetric particle geometries when locally exciting the particle nonsymmetrically, taking advantage of the highly confined electron beam. Extended to angle- and polarization-resolved schemes, this opened new venues in probing the field profiles, oscillation strengths and phases, and the light coupling properties of plasmon modes on the nanoscale. $^{17-21}$ Applying STEM-CL as a standard highresolution tool, any factors distorting or limiting the fidelity of angle- and wavelength-resolved CL measurements evidently have to be known. For example, the role of the substrate for $\mathrm{CL}$ emission has been characterized ${ }^{22}$ or for layered semiconductors the importance of considering waveguide modes or Cherenkov radiation has been emphasized. ${ }^{23}$ In this Letter, we show that the symmetry breaking due to the propagation direction of the electron beam itself can ultimately limit the spectral data interpretation. As the electrons pass perpendicularly through a plasmonic silver nanodisk, slightly different charge densities are excited on both disk faces, thus generating a dipole moment aligned with the electron trajectory. Spectrally overlapping the plasmonic radial breathing mode (RBM) of the nanodisk, the far field interference of the CL patterns of these two modes leads to a distinct spectral shift of about $8 \mathrm{~nm}$ in the backward and forward directions with respect to the beam trajectory. Importantly, this effect is also present when symmetrically exciting a geometrically symmetric particle. Our experimental results are supported by numerical simulations that we also apply to illustrate the individual role of the involved parameters. Although demonstrated here for a specific nanodisk geometry, the effect might be noticeable in a wide range of nanooptical systems featuring multiple

Received: October 12, 2020

Revised: November 27, 2020

Published: December 18, 2020 


\section{a)}
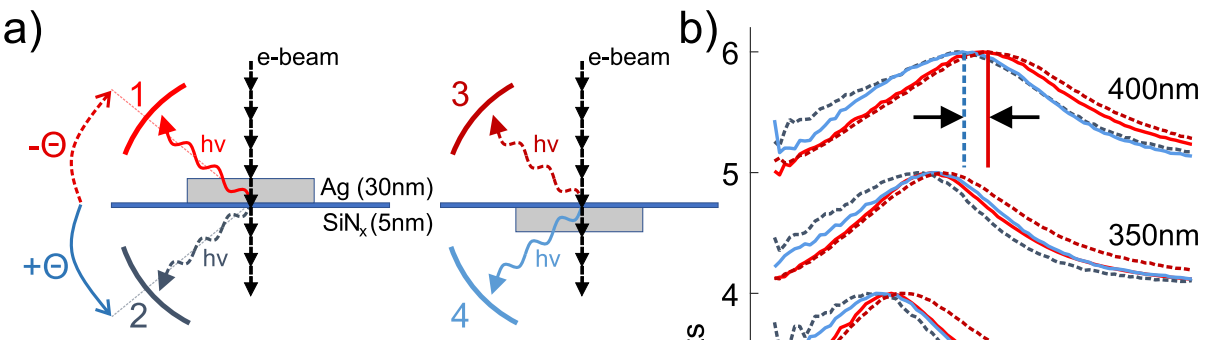

c)
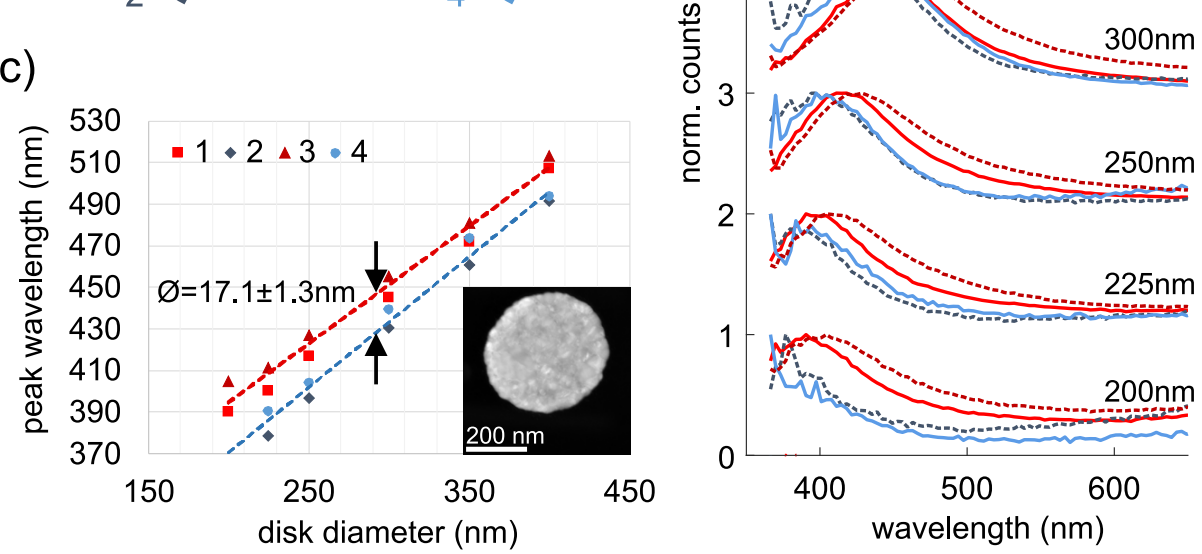

Figure 1. Experimental two-mirror CL spectroscopy. (a) Sketch of the measurement setup: A $200 \mathrm{keV}$ electron beam traverses a silver nanodisk (200-400 nm diameter) on a $5 \mathrm{~nm}$ thick $\mathrm{SiN}_{x}$ substrate along its symmetry axis, exciting the RBM. CL is collected by two mirrors above (red) and below (blue) the sample for both orientations of the substrate, corresponding to the sequentially measured spectra $1-4$. (b) Normalized CL spectra acquired in backward (red) and forward directions (blue). The solid lines indicate detection facing the particle, while the dashed lines correspond to detection through the substrate. (c) Peak positions from the spectra in (b) plotted versus disk diameter. The red data points are clearly spectrally shifted with respect to the blue ones, as emphasized by the linear fits and the black arrows.

excitations and should therefore be taken into account in highprecision CL experiments.

We prepared silver nanodisks of $30 \mathrm{~nm}$ thickness on a $5 \mathrm{~nm}$ thick $\mathrm{SiN}_{x}$ membrane by electron beam lithography, high vacuum metal deposition, and lift-off. The disk diameter was varied between 200 and $400 \mathrm{~nm}$; for a sample overview, see Figure S1 in Supporting Information. The CL experiments were performed on a FEI Tecnai F20 at $200 \mathrm{kV}$, equipped with a Gatan VULCAN CL spectrometer, consisting of two mirrors located above and below the sample, allowing the detection of the upward and downward emission of light separately, as schematically shown in Figure 1a. The simulations were based on the boundary element method, applying the MNPBEM toolbox. $^{24}$ The dielectric function of silver was taken from tabulated data. ${ }^{25,26}$ For further details, see Methods.

We excite the RBM mode by an electron beam transversing the nanodisk perpendicularly along its symmetry axis. ${ }^{27}$ While the RBM is a dark mode, that is, a mode with a zero net dipole moment, retardation lifts the dark character, giving rise to sizedependent CL emission. ${ }^{9}$ For the RBM, light is emitted around the $\pm 45^{\circ}$ directions with respect to the disk plane. ${ }^{28} \mathrm{CL}$ is detected by two mirrors that collect light in the backward (1) and forward (2) directions, as illustrated in Figure 1a, over polar angle ranges of $\left[-21^{\circ},-61^{\circ}\right]$ and $\left[+19^{\circ},+63^{\circ}\right]$, respectively, when defining the sample plane direction as $0^{\circ}$ and the electron beam direction as $90^{\circ}$. The mirrors focus the CL light onto glass fibers which are coupled to a spectrophotometer. Although the CL spectra 1 and 2 have to be acquired consecutively (acquisition time around $15 \mathrm{~s}$, see Methods), the CL system stability allows us to exclude any relevant instrumental signal drifts. However, increasing sample contamination from measurement to measurement due to carbon deposition plays a role, as discussed below. To probe the role of the orientation of the $\mathrm{SiN}_{x}$ substrate, the sample holder is removed from the STEM, and the sample is turned upside down and reinserted into the microscope to repeat the measurements on the same nanodisk, but with a reversed substrate orientation, corresponding to the measurements backward (3) and forward (4), as sketched in Figure 1a.

Figure $1 \mathrm{~b}$ shows the $\mathrm{CL}$ spectra from silver disks with diameters of $200-400 \mathrm{~nm}$ in the backward (1, 3; red) and forward (2, 4; blue) directions. All spectra clearly show the RBM peak, which redshifts with increasing disk diameter. ${ }^{27}$ This is accompanied by an increase of the peak width, due to enhanced radiation damping for larger disks. The measured peak positions are summarized in Figure 1c, together with linear fits that yield an average spectral shift between backward and forward CL peaks of approximately $17 \mathrm{~nm}$. For a detailed discussion of the peak position evolution and the error estimation, see Methods. We find in addition a quite small but significant redshift between spectra 1 and 3 (red squares and triangles in Figure 1c) and spectra 2 and 4 (blue circles and tilted squares). As the measurements were done consecutively in the order 1-2-4-3, electron-beam-induced contamination causing a redshift due to increasing the permittivity of the disk surrounding because of carbon deposition is the most likely cause. $^{29}$ The simulation results discussed below confirm this assumption. It is important to note that the substrate, $5 \mathrm{~nm}$ thin, shows no discernible influence on the CL peak position.

Next, we simulate the CL spectra with the MNPBEM toolbox. ${ }^{24}$ To simplify the problem, we neglect the substrate (which seems to play no important role in our system; for further discussion, see Figure S2 in the Supporting Information) yet take into account the limited angular range of the experiment by summing the CL intensity in the $\theta$ ranges $\left[-60^{\circ},-20^{\circ}\right]$ and $\left[+20^{\circ},+60^{\circ}\right]$. Figure $2 \mathrm{~b}$ shows the simulated 
a)

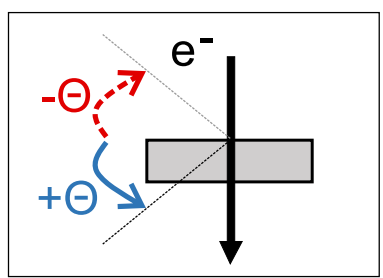

c)

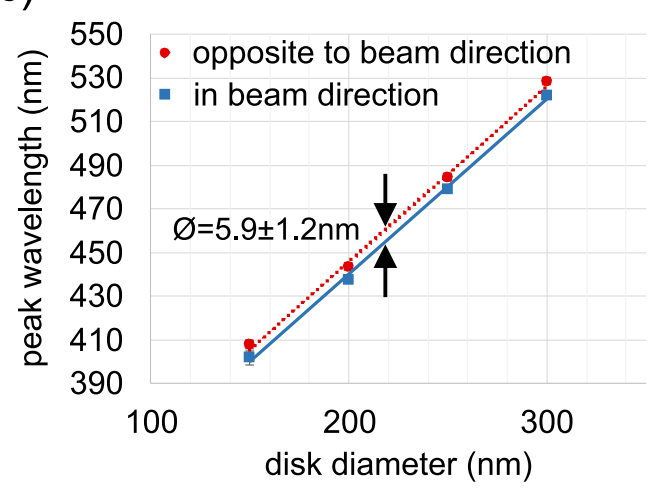

b)

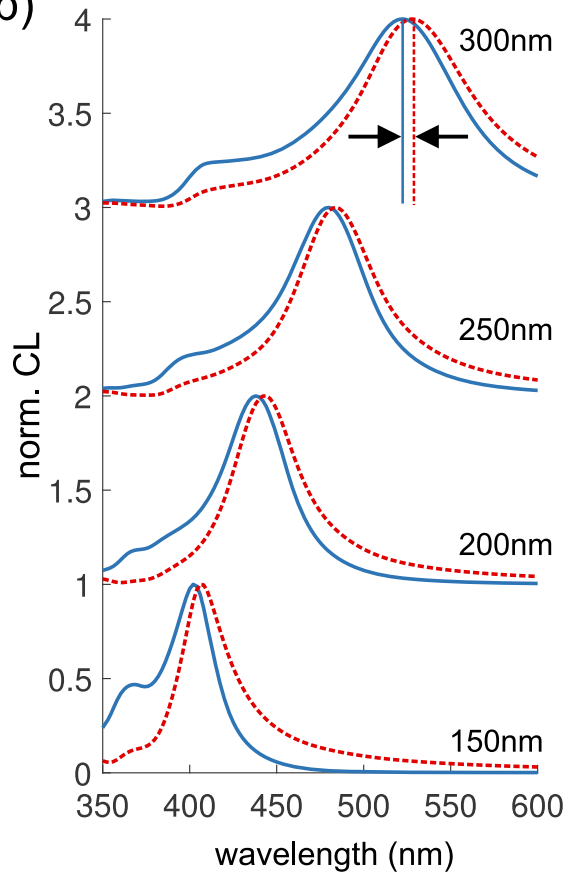

Figure 2. Simulated CL spectra. (a) Sketch of the excitation geometry: A $200 \mathrm{keV}$ electron beam traverses a $30 \mathrm{~nm}$ thick silver nanodisk along its symmetry axis, exciting the RBM. (b) CL spectra from the RBM for disk diameters of $150-300 \mathrm{~nm}$, integrated over the $\left[-60^{\circ},-20^{\circ}\right]$ and $\left[+20^{\circ}\right.$, $\left.+60^{\circ}\right]$ angular ranges. The main peak is attributed to the RBM, whereas the second peak on the left side of the main peak originates from the radially excited breathing mode $\mathrm{RBM}^{*}$. (c) Gaussian fitted peak positions from (b) are plotted as a function of disk diameter, showing the spectral shift between forward and backward CL.
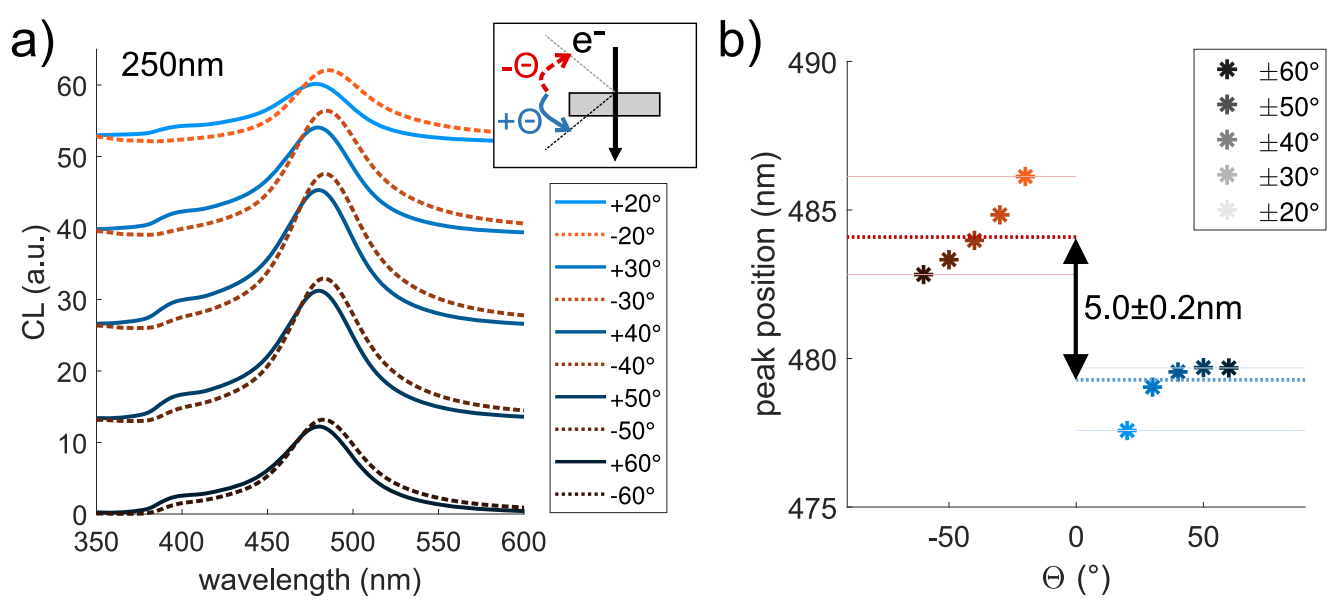

Figure 3. Simulated angular dependence of RBM CL. (a) CL spectra simulated for different emission angles from $\pm 60^{\circ}$ (bottom) to $\pm 20^{\circ}$ (top) of a silver nanodisk, $250 \mathrm{~nm}$ in diameter, and $30 \mathrm{~nm}$ thick. (b) Gaussian-fitted peak positions of the CL spectra in (a) versus emission angle. The dotted lines and the arrow show the peak position difference between the spectra in forward (integrated from $+20^{\circ}$ to $+60^{\circ}$ ) and in backward directions (integrated from $-20^{\circ}$ to $-60^{\circ}$ ).

spectra for disk diameters of 150-300 nm. Beside the diameter-dependent peak shift and broadening, we recover the RBM peak shift between the backward and forward spectra. The extracted peak values are plotted in Figure $2 c$, yielding an average peak shift of about $6 \mathrm{~nm}$. The considerable difference to the experimental result $(17 \mathrm{~nm})$ can be attributed to the contamination effect discussed above with possible further contributions from deviations in the simulated and experimental geometries and permittivities.

We now take a closer look at the angular dependence of the RBM CL emission from a $250 \mathrm{~nm}$ diameter nanodisk. Figure 3a displays simulated CL spectra for discrete $\theta$ values between $\pm 20^{\circ}$ and $\pm 60^{\circ}$. We find that (i) neither the peak wavelength nor the $\mathrm{CL}$ intensity is symmetric with respect to the particle plane $\left(\theta=0^{\circ}\right)$, (ii) the peak intensity is maximum around $45^{\circ}$, as expected for the $\mathrm{RBM}^{28}$ and (iii) the spectral peak shift increases for increasing deviations from the electron trajectory, that is, smaller values of $\theta$, as illustrated in Figure $3 \mathrm{~b}$. Further simulations shown in the Supporting Information illustrate that the spectral asymmetry increases with the thickness of the nanodisk (Figure S3), while it is practically independent of the disk diameter (Figure S4). Furthermore, the forward and backward CL peak positions are symmetrically shifted to higher and lower wavelengths with respect to the EELS peak 

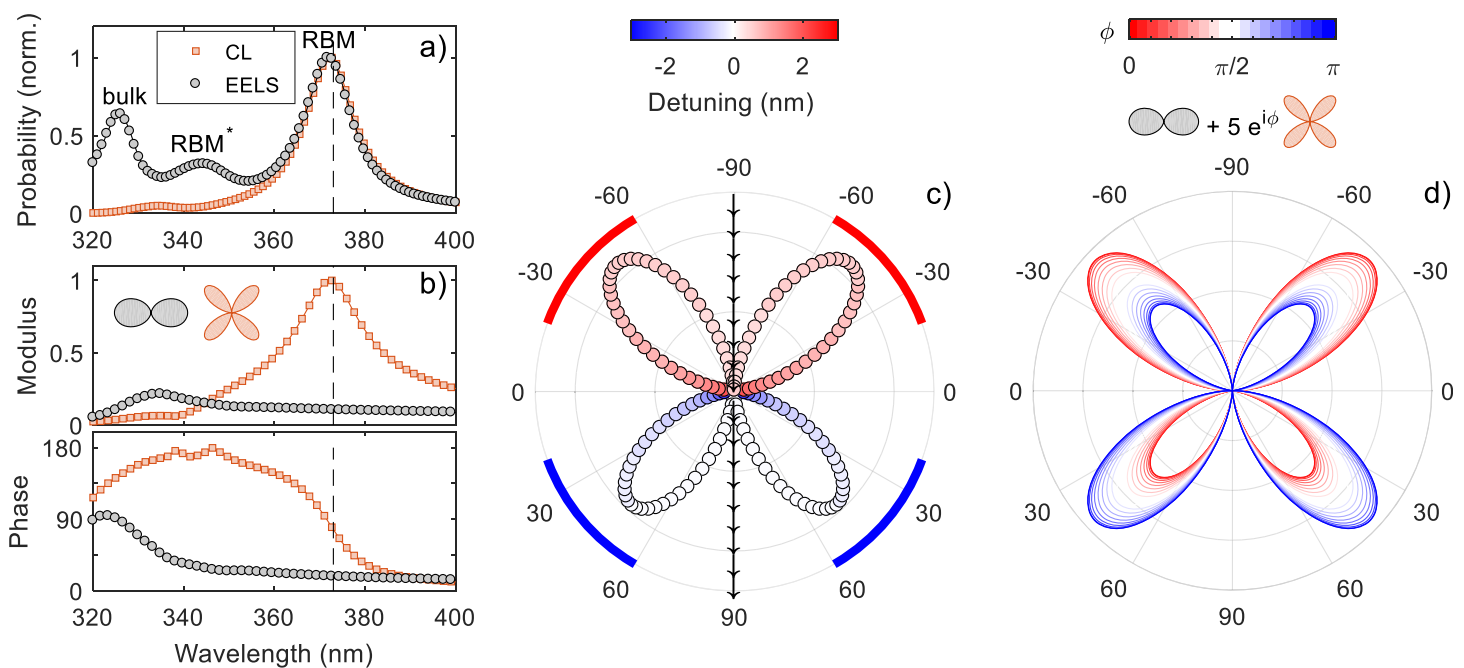

Figure 4. (a) Normalized simulated CL and EELS spectra for a silver nanodisk with a diameter of $200 \mathrm{~nm}$ and a thickness of $30 \mathrm{~nm}$. The EELS spectra show three pronounced peaks, which are attributed to the radial breathing mode (RBM), a radially excited breathing mode (RBM*), and bulk losses. (b) Multipole decomposition of the CL in the far field. We show the modulus and phase of the multipole coefficients for the vertical dipole (black circles) and the quadrupole (red squares). (c) Polar plot of emitted CL. The radii scale with the maxima of the spectra emitted in a given spatial direction, and the colors encode the detunings of the wavelengths at the maxima with respect to the RBM peak maximum at $373 \mathrm{~nm}$ given in (a). The red and blue arcs indicate the directions covered by the collecting mirrors in the experiment. (d) Emission pattern of generic vertical dipole and quadrupole fields for different phase relations $\phi$ between the fields, see eq 2. For $\phi=0(\phi=\pi)$ the CL emission into the backward (forward) direction dominates.

(Figure S5). Finally, the peak intensities and angles of maximum emission are plotted in Figure S6. To summarize so far, we find a spectral shift between the forward and backward CL emission from silver nanodisks that is not due to the presence of the substrate, carbon contamination, or the intrinsic angular dependence of the RBM emission. We thus conclude that the underlying reason is symmetry breaking due to the electron trajectory itself, a claim that we will now substantiate.

The electron beam approaching the nanodisk along its symmetry axis excites the RBM on both disk faces. The symmetry breaking due to the electron propagation direction leads to different charge densities on both sides of the disk, inducing a vertical dipole moment. Indeed, the corresponding spectral peaks are retrieved by CL spectra simulated around the direction normal to the electron trajectory (dashed arrows in Figure S7), while they are absent for larger angles (Figure S8), as expected for a vertically oriented dipole (Supporting Information). The spectral signatures of this mode are as well evident in Figures S3c and S4c, marked there by the red circles. For a more quantitative analysis, we take from our simulation results (CL and EELS spectra in Figure 4a) the scattered CL fields far away from the nanodisk and perform a multipole decomposition, following the procedure described in detail in Chapter 9 of Jackson. ${ }^{30}$ For the scattered magnetic field along direction $n$ we obtain

$$
\begin{aligned}
\boldsymbol{H}_{\mathrm{sca}}(\boldsymbol{r}) \rightarrow \frac{e^{i k r}}{k r} \sum_{l, m}(-i)^{l+1} \\
\\
{\left[a_{\mathrm{E}}(l, m) \boldsymbol{X}_{l m}+a_{\mathrm{M}}(l, m) \boldsymbol{n} \times \boldsymbol{X}_{l m}\right] }
\end{aligned}
$$

with a similar expression for the scattered electric field. $k$ is the wavenumber of light, $l$ and $m$ are the angular degree and order, respectively, and $\boldsymbol{X}_{l m}$ is the vector spherical harmonics characterizing the angular distribution of the scattered fields. Importantly, the above decomposition can be performed for nanoparticles of arbitrary size and the entire field distribution is described by the electric and magnetic multipole coefficients $a_{\mathrm{E}}(l, m)$ and $a_{\mathrm{M}}(l, m)$. From our simulation results, we find that for the electron beam propagating along the symmetry axis of the nanodisk only multipoles with $m=0$ contribute to eq 1 , and over a large spectral range the scattered fields are governed by

$$
\boldsymbol{H}_{\mathrm{sca}}(\boldsymbol{r}) \rightarrow \frac{e^{i k r}}{k r}\left[(-i)^{2} a_{\mathrm{E}}(1,0) \boldsymbol{X}_{10}+(-i)^{3} a_{\mathrm{E}}(2,0) \boldsymbol{X}_{20}\right]
$$

where the coefficient $a_{\mathrm{E}}(1,0)$ accounts for a vertical electric dipole and $a_{\mathrm{E}}(2,0)$ for an electric quadrupole. The spectral dependence of the modulus and phase of the coefficients is shown in Figure $4 \mathrm{~b}$. The dipole coefficient $a_{\mathrm{E}}(1,0)$ (circles) has no significant wavelength dependence, at least for wavelengths above $340 \mathrm{~nm}$, indicating that the symmetry breaking of the electron beam excitation is not significantly enhanced by plasmon resonances. This broadband excitation is similar to the transition radiation and surface plasmon emission of flat metal films. ${ }^{2}$ In contrast, the electric quadrupole coefficient $a_{\mathrm{E}}(2,0)$ (squares) exhibits a clear resonance behavior, regarding both modulus and phase, as expected for the CL emission of the RBM resonance mode. This is the important point: because of the resonance behavior of $a_{\mathrm{E}}(2,0)$, the dipole and quadrupole fields in eq 2 add up differently at different wavelengths. For red detuning with respect to the $\mathrm{RBM}$ resonance (i.e., the peak at $373 \mathrm{~nm}$ in Figure $4 \mathrm{a}$ corresponding to the $4 \pi$ integrated CL signal), more light is scattered in the direction antiparallel to the propagation direction of the electron beam (negative angles), see also Figure $4 \mathrm{~d}$, whereas for blue detuning the situation is reversed. As a result, the spectral maximum of the emitted radiation depends on the emission angle. This is shown in Figure $4 \mathrm{c}$ where we plot the red or blue detuning of the maximum of the spectra emitted into different directions. 
In conclusion, we have shown that the electron beam in STEM-CL can induce asymmetric surface charge densities giving rise to a dipole mode aligned with the electron trajectory. Even when spectrally well separated from a mode of interest (in our case the spectral peaks of the plasmonic vertical dipole and RBM are separated by $40 \mathrm{~nm}$ ), their far-field interference can lead to direction-dependent significant spectral shifts. We thus face an ultimate limit to the achievable fidelity in CL measurements on systems involving multiple excitations. This should evidently be considered in highprecision experiments, together with a choice of optimized operation parameters, where appropriate.

\section{METHODS}

Sample Preparation. Silver nanodisks were prepared by electron beam lithography on a $5 \mathrm{~nm}$ thin $\mathrm{SiN}_{x}$ membrane. The membrane was plasma cleaned for $10 \mathrm{~s}$ in air plasma prior to resist deposition (poly(methyl)methacrylate) and baking at $180{ }^{\circ} \mathrm{C}$ for $5 \mathrm{~min}$. After electron beam exposure in a RAITH eLINE+ system at $20 \mathrm{kV}$ and $33 \mathrm{pA}$, the sample was developed and $30 \mathrm{~nm}$ silver was thermally evaporated at a rate $1 \mathrm{~nm} / \mathrm{s}$ followed by a standard lift-off in acetone at $40{ }^{\circ} \mathrm{C}$.

Cathodoluminescence. CL experiments were performed on the TEM FEI Tecnai F20 equipped with the Gatan VULCAN CL spectrometer. The spectrometer consists of two mirrors. One is located above the sample covering the collection angles ranging from $-61^{\circ}$ to $-21^{\circ}$ and one is located below the sample covering the collection angles ranging from $+19^{\circ}$ to $+63^{\circ}$ when measured from the sample plane. This allows the detection of the upward and downward emission of light separately. The azimuthal angle of both mirrors varies between $-\pi / 2$ to $+\pi / 2$. The microscope was operated at $200 \mathrm{kV}$ in scanning regime with the electron beam current of $8 \mathrm{nA}$. CL linescans across the disk were acquired with an exposure time of $1.5 \mathrm{~s}$ per pixel, resulting in acquisition times of 13.5 to $18 \mathrm{~s}$ for the spectra shown in Figure 1 averaged over the center pixels of the disks.

Data Processing. The spectra shown in Figure 1a were first intensity corrected, accounting for the wavelengthdependent sensitivity of the mirrors, optical fiber, grating, and CCD camera. ${ }^{31}$ After normalization of each spectrum by diving through its acquisition time, a reference spectrum, acquired close to the disk on the bare $\mathrm{SiN}_{x}$ substrate, was subtracted. It is important to note that this step strongly enhanced the data quality in terms of signal-to-noise ratio. Because of a systematic noise, most likely originating from the detection camera, we could reduce this contribution by just subtracting the reference, including no other signal. Finally the spectra were normalized to their maxima for better comparison of the RBM peak shifts (Figure $1 \mathrm{~b}$ ). The peak positions plotted in this manuscript were obtained by Gaussian fitting of the corresponding spectra, while the given error was related to twice the standard deviation. The energy split values given in Figure 3 and in Supporting Information are calculated from CL spectra, which where summed over the relevant angle range $\left(-60^{\circ}\right.$ to $-20^{\circ}$ and $+20^{\circ}$ to $+60^{\circ}$, respectively) before Gaussian fitting. The spectral shift of $17.1 \mathrm{~nm}$ given in Figure $1 \mathrm{c}$ is calculated from averaging the difference between red and blue mean values for each disk size. The red and blue mean values correspond to the arithmetic mean of 1 and 3 , and 2 and 4 , respectively, again for each disk size. The given error of \pm 1.3 $\mathrm{nm}$ is calculated from the fit errors, including error propagation. Carbon deposition during the experiment may lead to an additional redshift, which is a possible explanation for the observed shift between the red data points 1 and 3, and the blue data points 2 and 4 in the order of half of the spectral shift value mentioned above. This results in an effective spectral shift between forward and backward emission of approximately $8 \mathrm{~nm}$, close to the simulated value of $6 \mathrm{~nm}$.

Simulations. The CL experiments were modeled using the retarded solver of the MNPBEM toolbox, ${ }^{32}$ following the classical electrodynamics framework described in ref 2 . The electron beam was assumed to transverse the nanodisk along its symmetry axis, and the induced far-field was calculated at discrete orientation angles, characterized by both polar and azimuthal angles. The light intensity emitted toward a given polar angle was then calculated as the Poynting vector integrated over the azimuthal angles with constant polar angle. Calculations were performed using the relative permittivity of silver taken from ref 25 , for both nanodisks only and nanodisks sitting on top of a $\mathrm{SiN}_{x}$ membrane, except for data shown in Figure 4, where ref 26 was used, which highlights that the findings in this work are not limited by the chosen tabulated silver data. In case of nanodisks only (without substrate), the objects were embedded within an effective dielectric medium with constant relative permittivity ${ }^{33}$ of 1.7 , resulting in RBM peak energies in good agreement with the calculations that include the membrane with a relative permittivity of 4 (see Figure S2 in the Supporting Information). Only in case of Figure 4 we have chosen a relative permittivity of 1 , which shows the independence (embedded medium vs vacuum) on the discussed effect. Further details can be found in the methods section of ref 9 . The detection angle of the simulated CL spectra spanned over the range as indicted in the figure legends or captions, whereas in Figure $4 a, b$ the signal emitted in all directions $(4 \pi)$ was collected.

\section{ASSOCIATED CONTENT}

\section{Supporting Information}

The Supporting Information is available free of charge at https://pubs.acs.org/doi/10.1021/acs.nanolett.0c04084.

Overview of the EBL prepared silver nanodisks (Figure $\mathrm{S} 1$ ), influence of the substrate (Figure S2), influence of the disk thickness (Figure S3), influence of the disk diameter (Figure S4), comparing EELS and CL (Figure S5), CL peak intensities (Figure S6), CL emission to lower angles (Figure S7) and higher angles (Figure S8) (PDF)

\section{AUTHOR INFORMATION}

\section{Corresponding Author}

Franz-Philipp Schmidt - Institute of Physics, University of Graz, Graz 8010, Austria; (o orcid.org/0000-0003-01252704; Email: franz.schmidt@uni-graz.at

\section{Authors}

Arthur Losquin - Thales Research and Technology, Palaiseau 91767, France

Michal Horák - Central European Institute of Technology, Brno University of Technology, Brno 612 00, Czech Republic; o orcid.org/0000-0001-6503-8294

Ulrich Hohenester - Institute of Physics, University of Graz, Graz 8010, Austria 
Michael Stöger-Pollach - University Service Centre for Transmission Electron Microscopy, TU Wien, Wien 1040, Austria

Joachim R. Krenn - Institute of Physics, University of Graz, Graz 8010, Austria

Complete contact information is available at:

https://pubs.acs.org/10.1021/acs.nanolett.0c04084

\section{Notes}

The authors declare no competing financial interest.

\section{ACKNOWLEDGMENTS}

This work was supported by the FWF SFB NextLite (F4905N23), FWF under project P 31264, NAWI Graz, the Graz Centre for Electron Microscopy (ZFE), and the University Service Centre for Transmission Electron Microscopy (USTEM). This work was partially supported by European Union's Horizon 2020 program (project SINNCE, No. 810626). We thank Thomas Götsch, Christoph Pratsch, and Thomas Lunkenbein for fruitful discussions on electron-matter interaction and carbon contamination in transmission electron microscopes. M.H. acknowledges the support of Thermo Fisher Scientific and CSMS scholarship 2019.

\section{DEDICATION}

Dedicated to the memory of Mark Stockman. We are grateful to Mark Stockman who, at the Nano \& Photonics Meeting in Mauterndorf in 2013, first dismissed our dark plasmonic breathing mode (of course) but finally approved it. We will always miss his vision and passion for the field of plasmonics.

\section{REFERENCES}

(1) Yamamoto, N.; Araya, K.; García de Abajo, F. J. Photon emission from silver particles induced by a high-energy electron beam. Phys. Rev. B 2001, 64, 1069.

(2) García de Abajo, F. J. Optical excitations in electron microscopy. Rev. Mod. Phys. 2010, 82, 209-275.

(3) Kociak, M.; Gloter, A.; Stéphan, O. A spectromicroscope for nanophysics. Ultramicroscopy 2017, 180, 81-92.

(4) Losquin, A.; Lummen, T. T. A. Electron microscopy methods for space-, energy-, and time-resolved plasmonics. Front. Phys. 2017, 12, 127301.

(5) Kociak, M.; Stéphan, O. Mapping plasmons at the nanometer scale in an electron microscope. Chem. Soc. Rev. 2014, 43, 38653883.

(6) Losquin, A.; Kociak, M. Link between Cathodoluminescence and Electron Energy Loss Spectroscopy and the Radiative and Full Electromagnetic Local Density of States. ACS Photonics 2015, 2, $1619-1627$

(7) Losquin, A.; Zagonel, L. F.; Myroshnychenko, V.; RodríguezGonzález, B.; Tencé, M.; Scarabelli, L.; Förstner, J.; Liz-Marzán, L. M.; García de Abajo, F. J.; Stéphan, O.; Kociak, M. Unveiling nanometer scale extinction and scattering phenomena through combined electron energy loss spectroscopy and cathodoluminescence measurements. Nano Lett. 2015, 15, 1229-1237.

(8) Kawasaki, N.; Meuret, S.; Weil, R.; Lourenço-Martins, H.; Stéphan, O.; Kociak, M. Extinction and Scattering Properties of HighOrder Surface Plasmon Modes in Silver Nanoparticles Probed by Combined Spatially Resolved Electron Energy Loss Spectroscopy and Cathodoluminescence. ACS Photonics 2016, 3, 1654-1661.

(9) Schmidt, F.-P.; Losquin, A.; Hofer, F.; Hohenau, A.; Krenn, J. R.; Kociak, M. How Dark Are Radial Breathing Modes in Plasmonic Nanodisks? ACS Photonics 2018, 5, 861-866.
(10) Gómez-Medina, R.; Yamamoto, N.; Nakano, M.; García de Abajo, F. J. Mapping plasmons in nanoantennas via cathodoluminescence. New J. Phys. 2008, 10, 105009.

(11) Chaturvedi, P.; Hsu, K. H.; Kumar, A.; Fung, K. H.; Mabon, J. C.; Fang, N. X. Imaging of plasmonic modes of silver nanoparticles using high-resolution cathodoluminescence spectroscopy. ACS Nano 2009, 3, 2965-2974.

(12) Kumar, A.; Fung, K. H.; Fang, N. X. Mapping of surface plasmon polaritons on nanostructured thin film disks using cathodoluminescence imaging. Photonic and Phononic Properties of Engineered Nanostructures 2011, 79461U.

(13) Atre, A. C.; Brenny, B. J. M.; Coenen, T.; García-Etxarri, A.; Polman, A.; Dionne, J. A. Nanoscale optical tomography with cathodoluminescence spectroscopy. Nat. Nanotechnol. 2015, 10, 429436.

(14) Coenen, T.; Haegel, N. M. Cathodoluminescence for the 21st century: Learning more from light. Appl. Phys. Rev. 2017, 4, No. 031103.

(15) Kociak, M.; Zagonel, L. F. Cathodoluminescence in the scanning transmission electron microscope. Ultramicroscopy 2017, $176,112-131$.

(16) Hancu, I. M.; Curto, A. G.; Castro-Lopez, M.; Kuttge, M.; van Hulst, N. F. Multipolar Interference for Directed Light Emission. Nano Lett. 2014, 14, 166-171.

(17) Coenen, T.; Bernal Arango, F.; Femius Koenderink, A.; Polman, A. Directional emission from a single plasmonic scatterer. Nat. Commun. 2014, 5, 3250.

(18) Coenen, T.; Polman, A. Optical Properties of Single Plasmonic Holes Probed with Local Electron Beam Excitation. ACS Nano 2014, 8, 7350-7358.

(19) Yamamoto, N. Development of high-resolution cathodoluminescence system for STEM and application to plasmonic nanostructures. Microscopy (Oxford, U. K.) 2016, 65, 282-295.

(20) Thollar, Z.; Wadell, C.; Matsukata, T.; Yamamoto, N. Sannomiya, T. Three-Dimensional Multipole Rotation in Spherical Silver Nanoparticles Observed by Cathodoluminescence. ACS Photonics 2018, 5, 2555-2560.

(21) Matsukata, T.; Wadell, C.; Matthaiakakis, N.; Yamamoto, N.; Sannomiya, T. Selected Mode Mixing and Interference Visualized within a Single Optical Nanoantenna. ACS Photonics 2018, 5, 49864992.

(22) Fiedler, S.; Raza, S.; Ai, R.; Wang, J.; Busch, K.; Stenger, N.; Mortensen, N. A.; Wolff, C. Importance of substrates for the visibility of "dark" plasmonic modes. Opt. Express 2020, 28, 13938-13948.

(23) Stöger-Pollach, M.; Bukvišová, K.; Schwarz, S.; Kvapil, M.; Šamořil, T.; Horák, M. Fundamentals of cathodoluminescence in a STEM: The impact of sample geometry and electron beam energy on light emission of semiconductors. Ultramicroscopy 2019, 200, 111124

(24) Hohenester, U.; Trügler, A. MNPBEM - A Matlab toolbox for the simulation of plasmonic nanoparticles. Comput. Phys. Commun. 2012, 183, 370-381.

(25) Palik, E. D. Handbook of optical constants of solids; Academic Press handbook series; Academic Press: Orlando, 1985.

(26) Johnson, P. B.; Christy, R. W. Optical Constants of the Noble Metals. Phys. Rev. B 1972, 6, 4370-4379.

(27) Schmidt, F.-P.; Ditlbacher, H.; Hohenester, U.; Hohenau, A.; Hofer, F.; Krenn, J. R. Dark plasmonic breathing modes in silver nanodisks. Nano Lett. 2012, 12, 5780-5783.

(28) Schaffernak, G.; Krug, M. K.; Belitsch, M.; Gasparic, M.; Ditlbacher, H.; Hohenester, U.; Krenn, J. R.; Hohenau, A. Plasmonic Dispersion Relations and Intensity Enhancement of Metal-InsulatorMetal Nanodisks. ACS Photonics 2018, 5, 4823-4827.

(29) Hörl, A.; Haberfehlner, G.; Trügler, A.; Schmidt, F.-P.; Hohenester, U.; Kothleitner, G. Tomographic imaging of the photonic environment of plasmonic nanoparticles. Nat. Commun. 2017, 8, 37.

(30) Jackson, J. D. Classical electrodynamics; 3rd ed.; Wiley: New York, 1999. 
(31) Stöger-Pollach, M.; Kachtík, L.; Miesenberger, B.; Retzl, P. Transition radiation in EELS and cathodoluminescence. Ultramicroscopy 2017, 173, 31-35.

(32) Hohenester, U. Simulating electron energy loss spectroscopy with the MNPBEM toolbox. Comput. Phys. Commun. 2014, 185, 1177-1187.

(33) Martin, J.; Kociak, M.; Mahfoud, Z.; Proust, J.; Gérard, D.; Plain, J. High-resolution imaging and spectroscopy of multipolar plasmonic resonances in aluminum nanoantennas. Nano Lett. 2014, $14,5517-5523$. 


\section{The Fundamental Limit of Plasmonic}

\section{Cathodoluminescence}

\section{Supporting Information}

Franz-Philipp Schmidt, ${ }^{*, \dagger}$ Arthur Losquin, ${ }^{\ddagger}$ Michal Horák, ${ }^{\circledR}$ Ulrich Hohenester, ${ }^{\dagger}$

Michael Stöger-Pollach, ${ }^{\S}$ and Joachim R. Krenn ${ }^{\dagger}$

$\dagger$ Institute of Physics, University of Graz, Austria

$\ddagger$ Thales Research and Technology, Palaiseau, France

\Central European Institute of Technology, Brno University of Technology, Czech Republic

$\S$ University Service Centre for Transmission Electron Microscopy, TU Wien, Austria

E-mail: franz.schmidt@uni-graz.at 


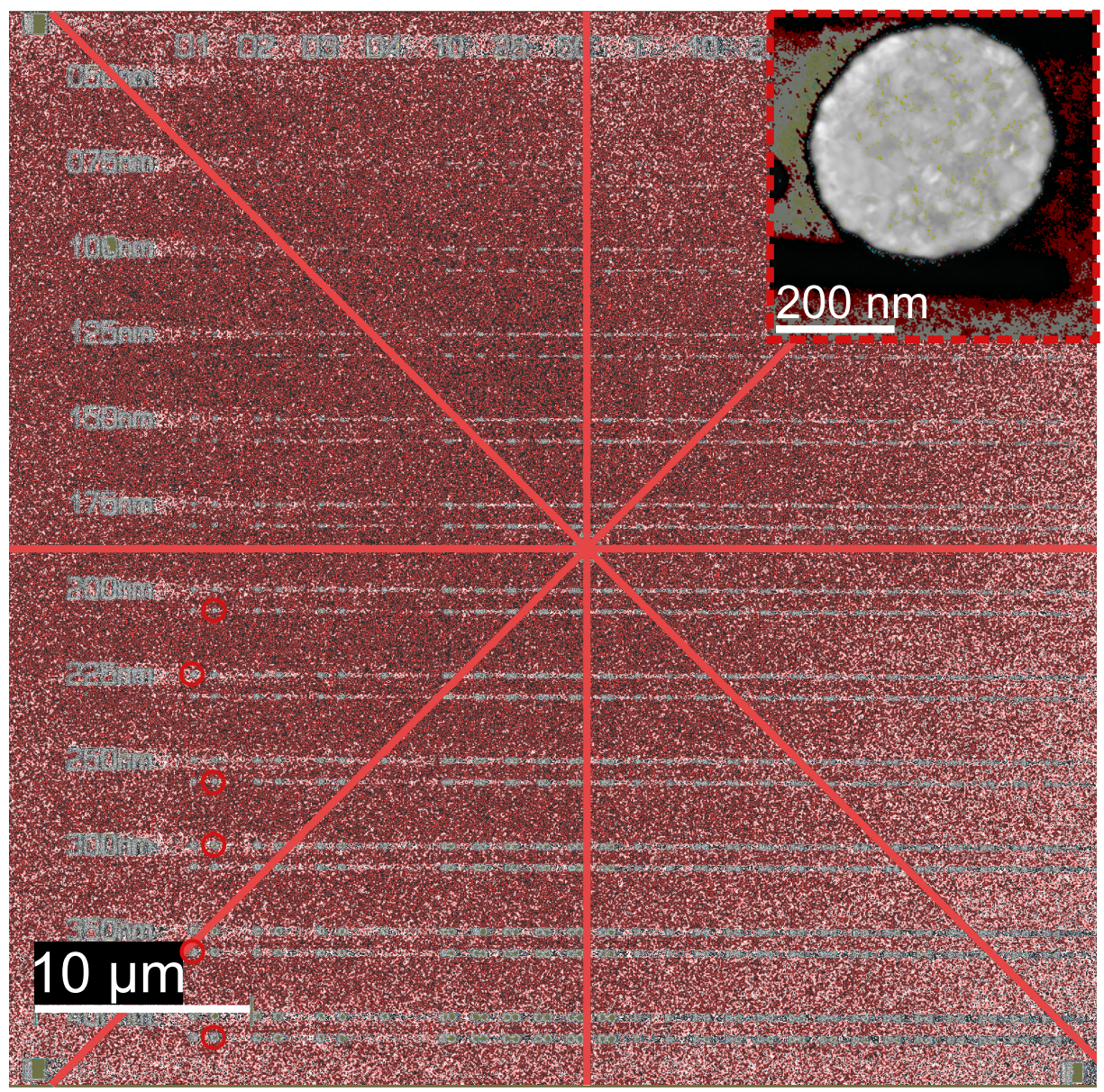

Figure S1: Overview SEM image of the EBL prepared silver nanodisks on a $5 \mathrm{~nm}$ thick $\mathrm{SiN}_{\mathrm{x}}$ substrate. The six disks with diameters of $200-400 \mathrm{~nm}$ which are studied in this paper are highlighted by red circles. A TEM high-angle annular dark field image of the $400 \mathrm{~nm}$ diameter disk is shown in the inset. 


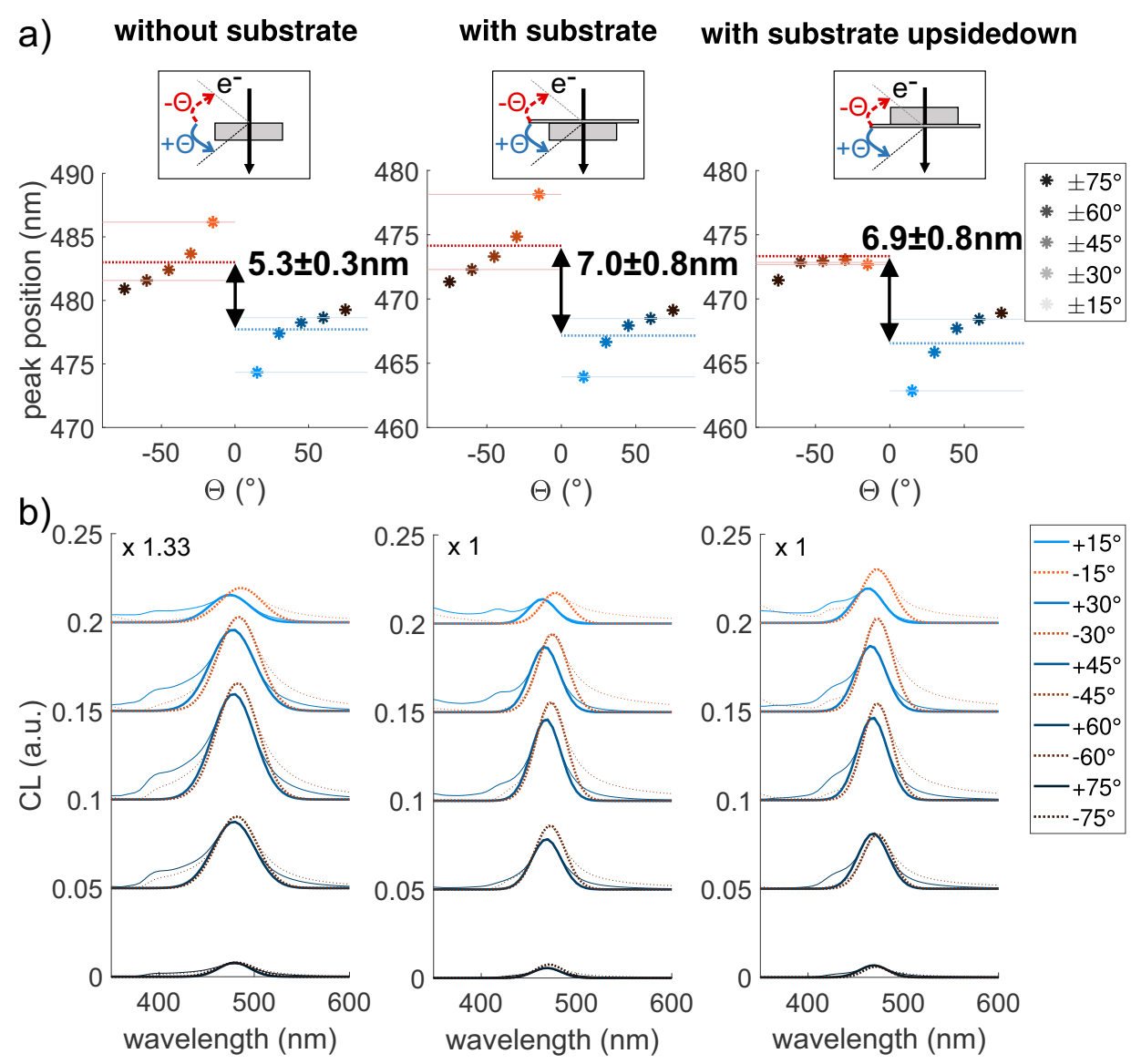

Figure S2: Simulation of the influence of the $5 \mathrm{~nm}$ thick $\mathrm{SiN}_{\mathrm{x}}$ substrate on the CL emission. a) Angle-resolved RBM peak positions of a silver nanodisk ( $250 \mathrm{~nm}$ diameter, $30 \mathrm{~nm}$ thickness) without substrate (left), with the substrate facing up (middle) and with the substrate facing down (right), as sketched in the insets. The dotted lines and the arrow show the peak position difference in forward (integrated from $+15^{\circ}$ to $+60^{\circ}$ ) and in backward directions (integrated from $-15^{\circ}$ to $-60^{\circ}$ ). b) Corresponding CL spectra at different angles, as given by the legend. The slight differences in the peak shifts, as given by the values in a), are not further considered as they are within or only slightly above the fit error. The higher peak splitting value of $5.3 \pm 0.3 \mathrm{~nm}$ in a) (left) compared to $5 \pm 0.2 \mathrm{~nm}$ in Figure 3, S3 and S4 is attributed to a different discretization in the simulation related to wavelength and angle $\left(\Delta \mathrm{x}=5.0 \mathrm{~nm}\right.$ and $\Delta \Theta=15^{\circ}$ instead of $\Delta \mathrm{x}=2 \mathrm{~nm}$ and $\left.\Delta \Theta=10^{\circ}\right)$. 

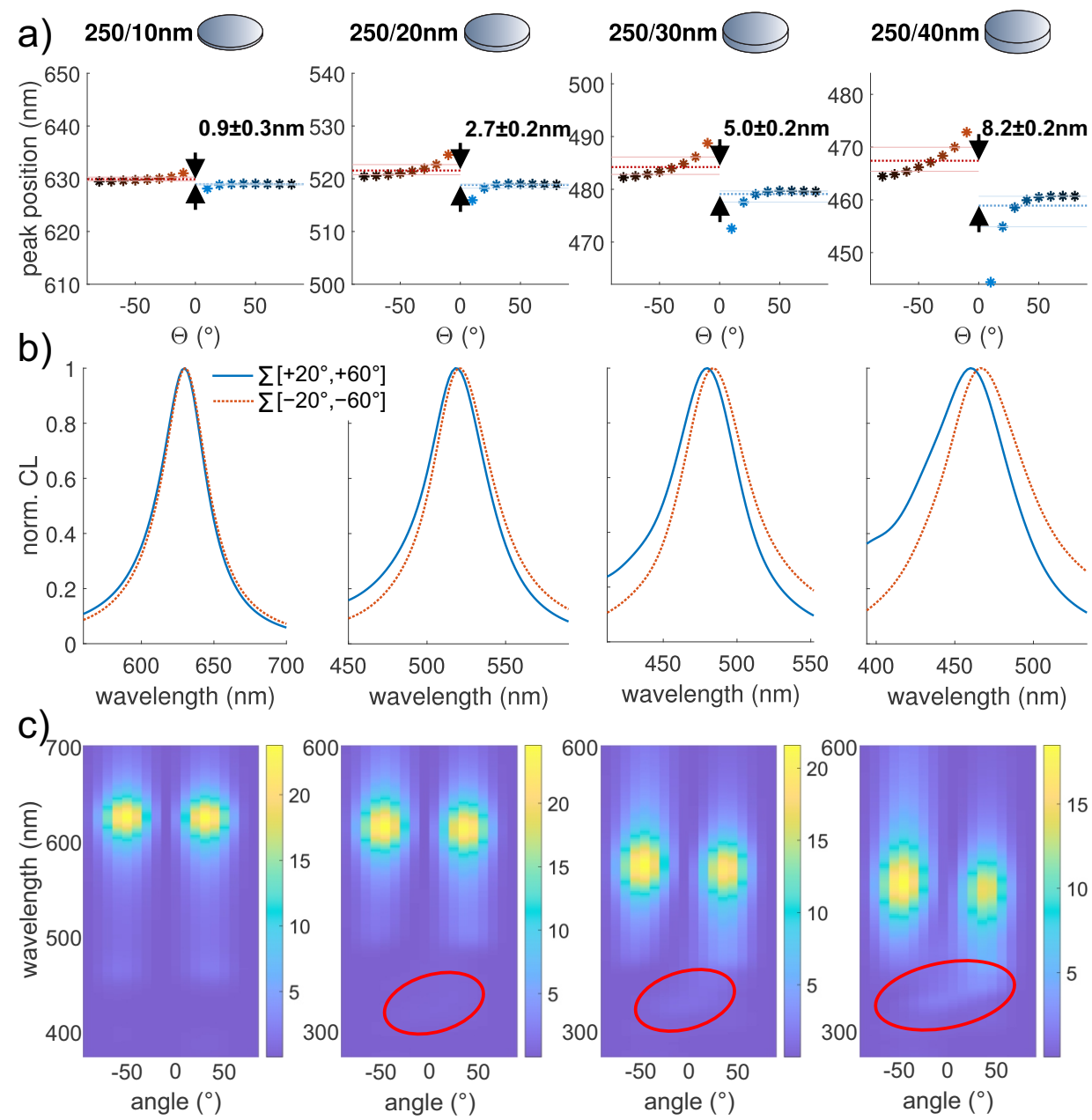

Figure S3: Simulation of the influence of the disk thickness on the CL emission. a) Angleresolved spectral RBM peak positions for disks of constant diameter $(250 \mathrm{~nm})$ and varying thickness of 10,20, 30 and $40 \mathrm{~nm}$. The shift was measured between the peak positions of the angle-averaged spectra shown in (b). b) Corresponding CL spectra, averaged over the angle ranges indicated in the legend. c) CL intensity vs. wavelength and emission angle, the bright spots correspond to the RBM peak maxima that show a mutual spectral shift increasing with disk thickness. The emission maxima appear close to $\pm 45^{\circ}$ in all cases, while the peak maxima shift generally to lower wavelengths for increasing thickness and thus increasing height/diameter ratio of the disks. The red circles mark the spectral feature of the vertical dipole mode. 

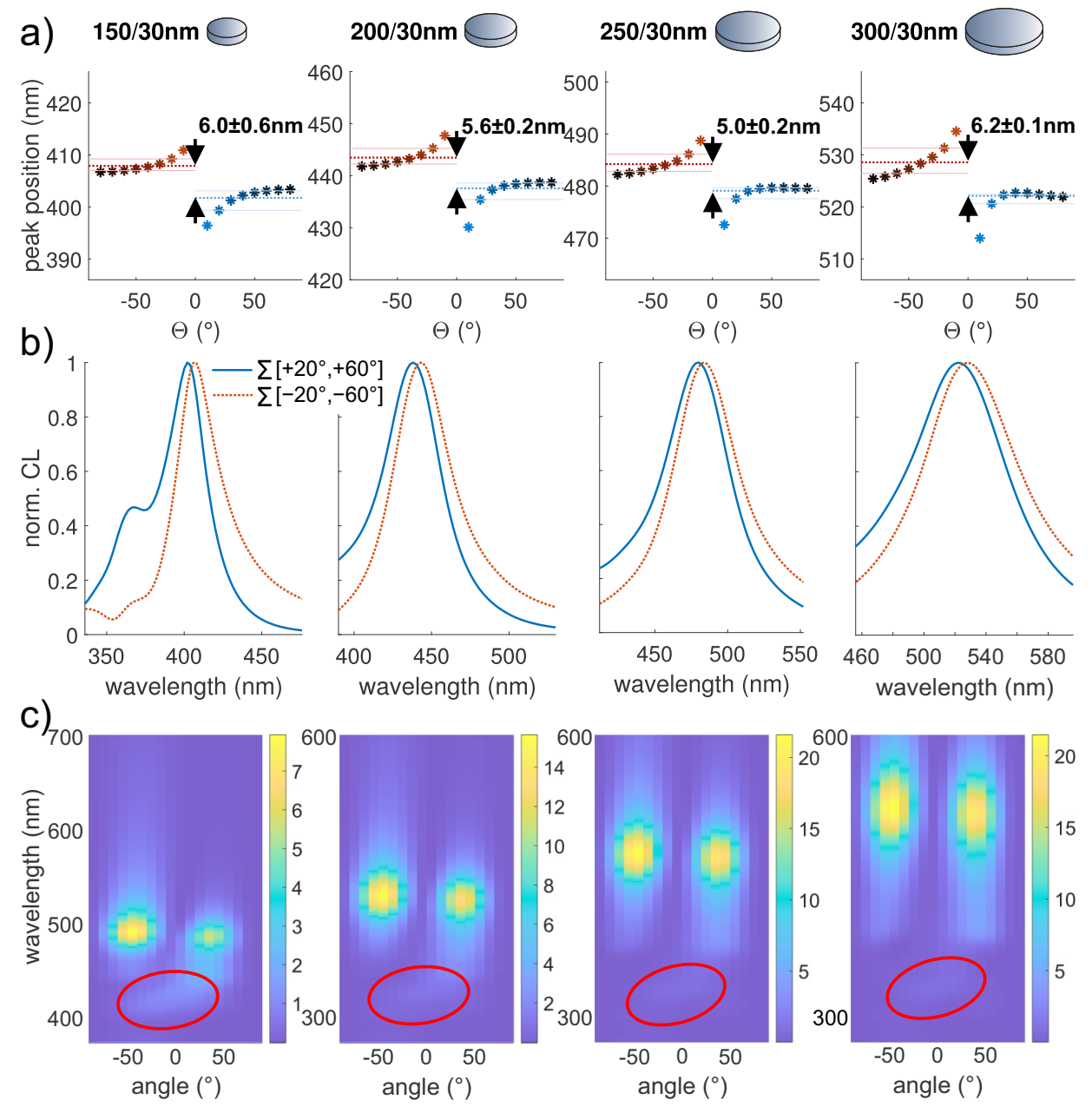

Figure S4: Simulation of the influence of the disk diameter on the CL emission. a) Angleresolved spectral RBM peak positions for disks of constant thickness $(30 \mathrm{~nm})$ and varying diameters of 150,200, 250 and $300 \mathrm{~nm}$. The shift was measured between the peak positions of the angle-averaged spectra shown in (b). b) Corresponding CL spectra, averaged over the angle ranges indicated in the legend. c) CL intensity vs. wavelength and emission angle, the bright spots correspond to the RBM peak maxima that show no marked trend for varying the disk diameters. The emission maxima appear close to $\pm 45^{\circ}$ in all cases, while the peak maxima shift to higher wavelengths for increasing diameters and thus decreasing height/diameter ratio of the disks. The red circles mark the spectral feature of the vertical dipole mode. 


\section{a)}
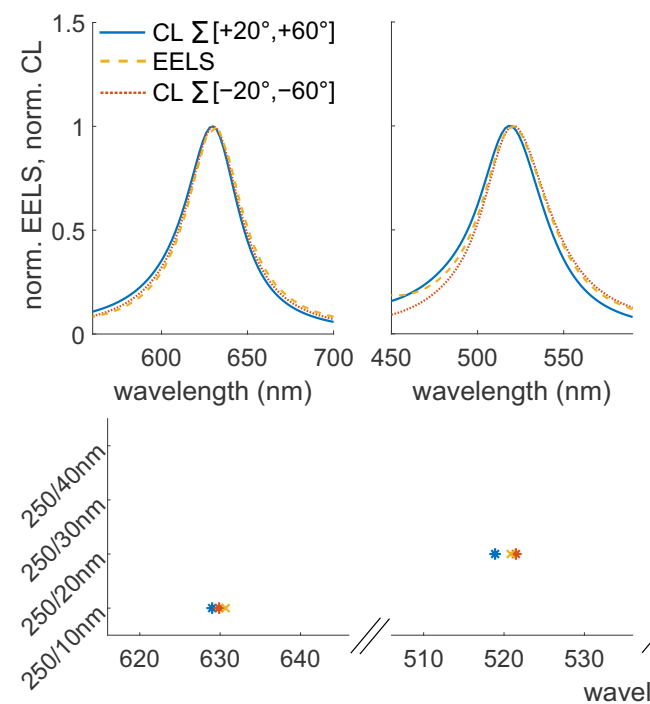

b) $150 / 30 \mathrm{~nm} \ominus$
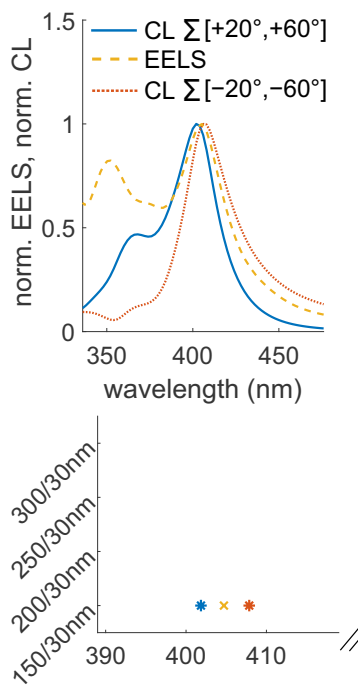

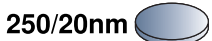

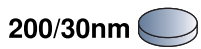

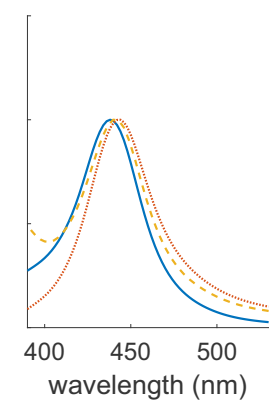

250/30nm

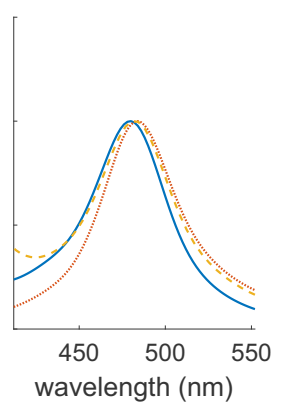

wavelength $(\mathrm{nm})$
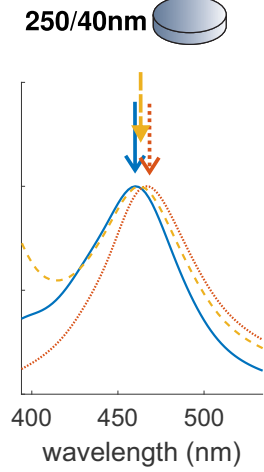
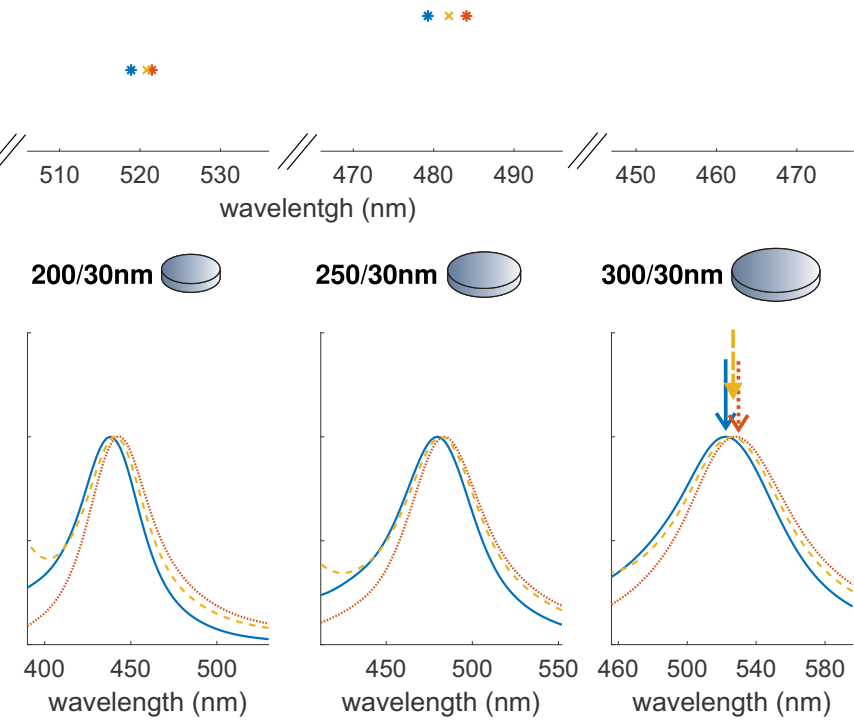

Figure S5: Comparing simulated CL and EELS spectra. a) Spectra for disks of constant diameter $(250 \mathrm{~nm})$ and varying thickness of 10, 20, 30 and $40 \mathrm{~nm}$ (top) and corresponding spectral peak positions (bottom). b) Spectra for disks of constant thickness (30 nm) and varying diameters of 150,200, 250 and $300 \mathrm{~nm}$ (top) and corresponding spectral peak positions (bottom). The CL spectra were averaged over the angle range indicated in the legend. 

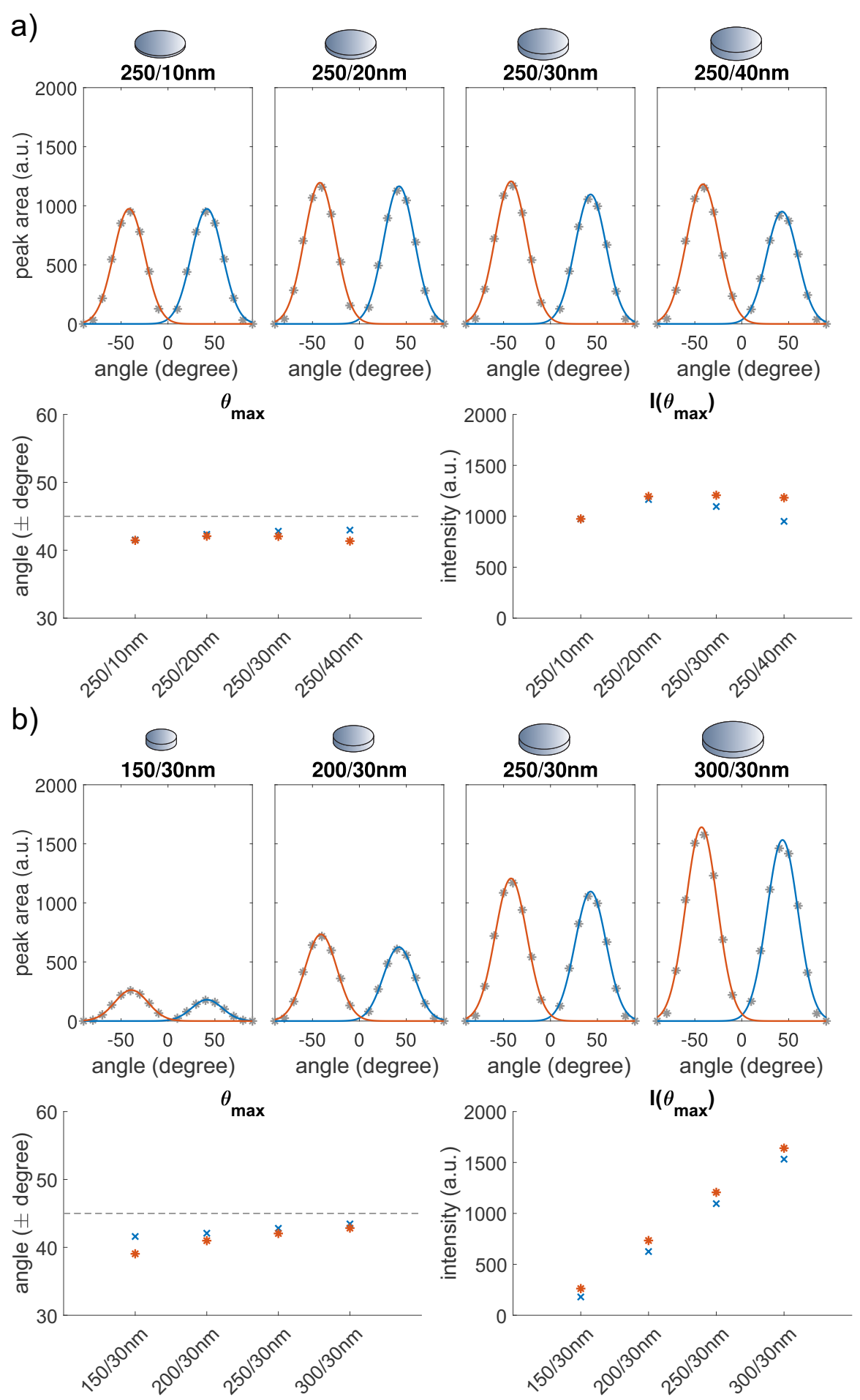

Figure S6: Simulated CL emission peak intensity. a) Fitted RBM peak areas (symbols) as function of the emission angle, for disks of constant thickness $(30 \mathrm{~nm})$ and varying diameters of 150, 200, 250 and $300 \mathrm{~nm}$ (top). The lines are Gaussian fits to the data points. Angles of maximum emission $\theta_{\max }$ (bottom left) and emission intensity in the $\theta_{\max }$ directions (bottom right), derived from the fit curves in the top panel. b) Same as in a) but for disks of constant thickness $(30 \mathrm{~nm})$ and varying diameters of 150, 200, 250 and $300 \mathrm{~nm}$. 

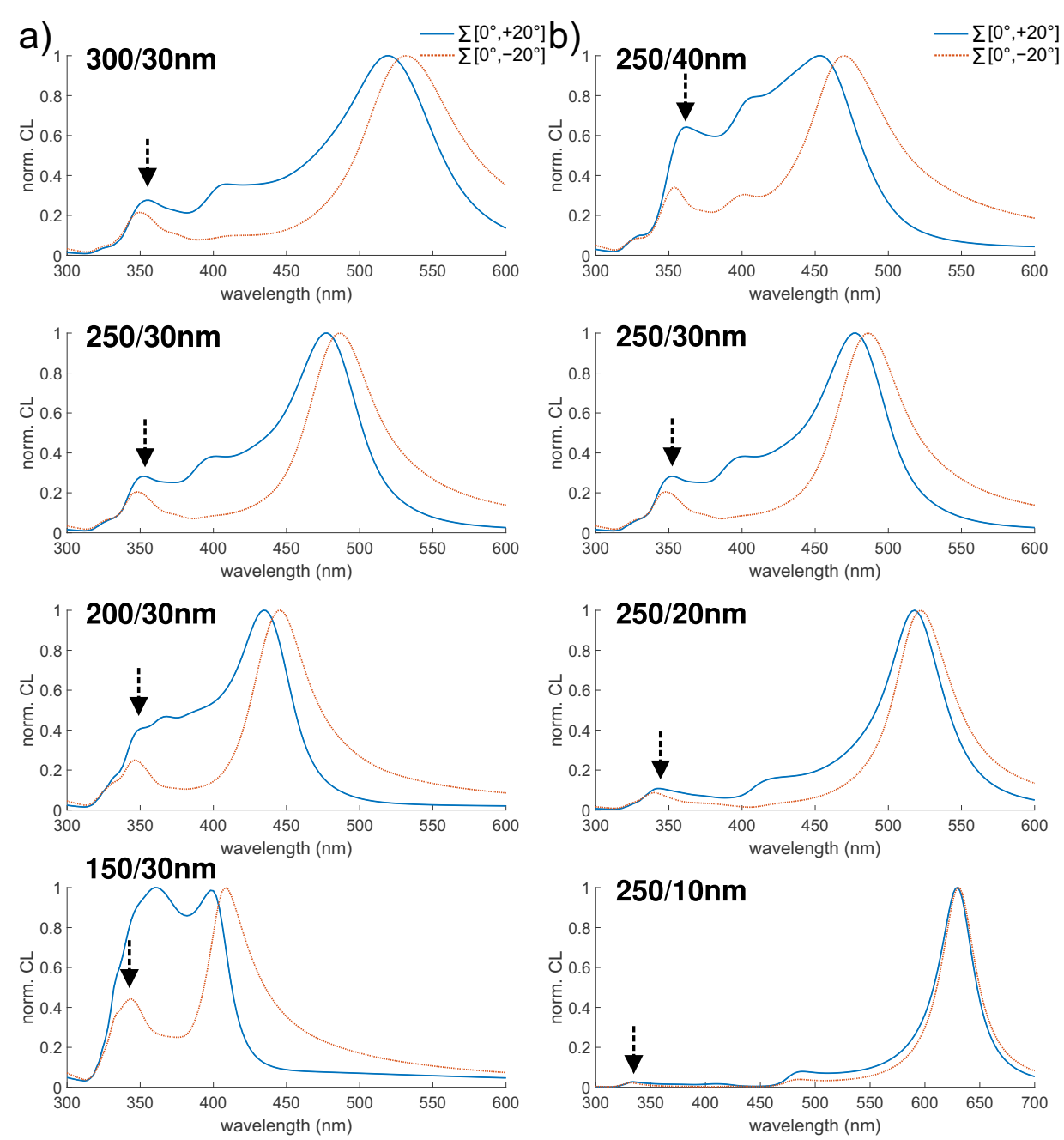

Figure S7: Simulated CL emission spectra over the spectral ranges $\left[0^{\circ}, \pm 20^{\circ}\right]$. The dashed arrows points to the vertical dipole mode. The varying disk diameter and thickness is indicted by the labels "diameter/thickness" $\mathrm{nm}$. The electron beam traverses along the symmetry axis of the disks. 

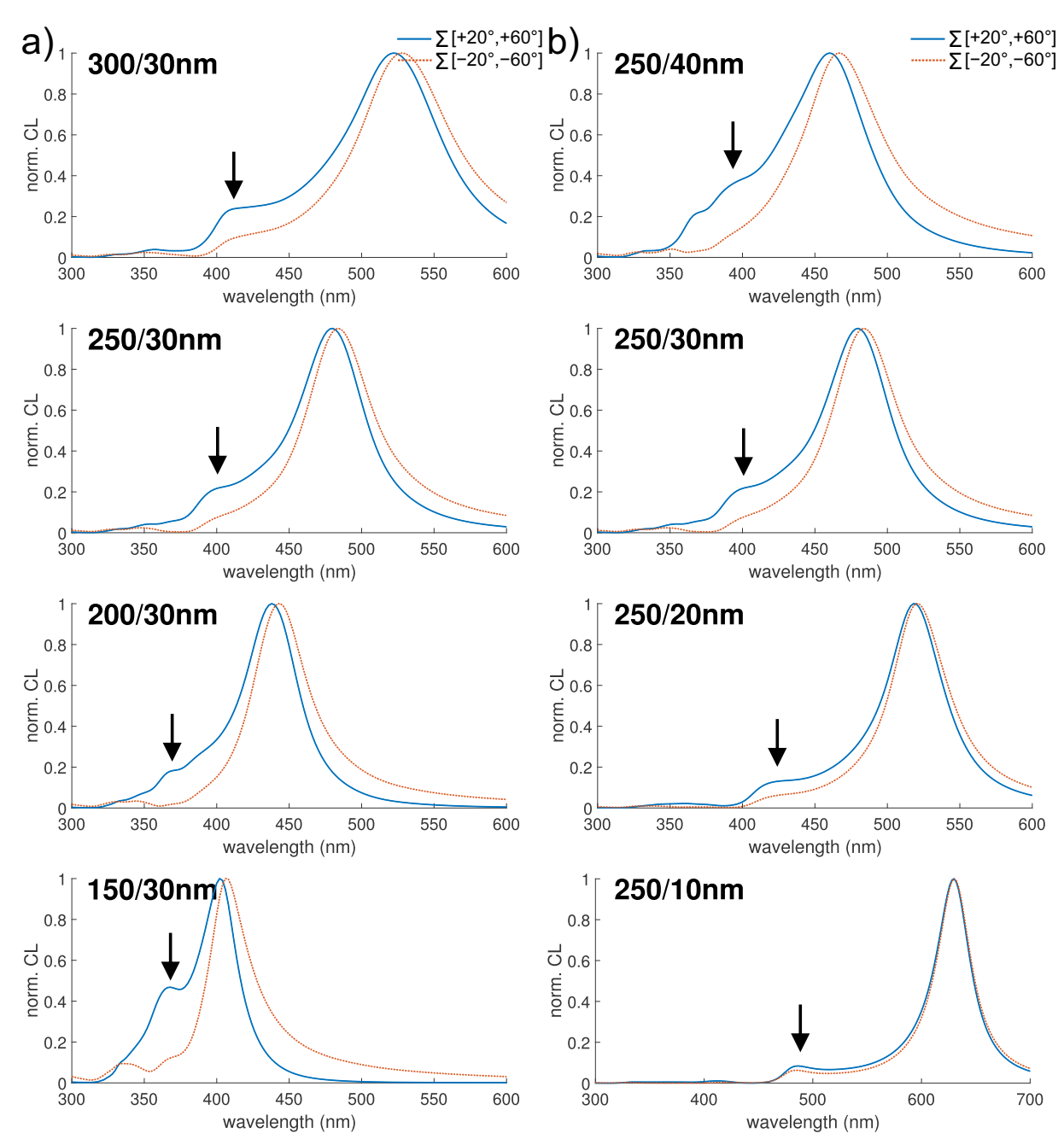

Figure S8: Simulated CL emission spectra over the spectral ranges $\left[ \pm 20^{\circ}, \pm 60^{\circ}\right]$. The solid arrows points to the $\mathrm{RBM}^{*}$ mode (radially excited breathing mode), while the vertical dipole at lower wavelength is hardly visible (compare with dashed arrows in Figure S7). The varying disk diameter and thickness is indicted by the labels "diameter/thickness" nm. The electron beam traverses along the symmetry axis of the disks. 